\title{
Induced production of BE-31405 by co-culturing of Talaromyces siamensis FKA-61 with a variety of fungal strains
}

\author{
Kenichi Nonaka ${ }^{1,2,3}$, Masato Iwatsuki ${ }^{1,2,3}$, Syunsuke Horiuchi ${ }^{1}$, Kazuro Shiomi ${ }^{1,2}$, Satoshi Ōmura ${ }^{1}$ \\ and Rokuro Masuma ${ }^{1,2}$
}

While isolating fungal strains from soil samples, two strains, Talaromyces siamensis FKA-61 and Phomopsis sp. FKA-62, were found to produce a black pigment near the boundary with an adjoining colony on agar plates. Moreover, strain FKA-61 inhibited the growth of strain FKA-62. Co-culture of $T$. siamensis FKA-61 with Phomopsis sp. FKA-62 resulted in the production of an antifungal agent, BE-31405, which proved active against Phomopsis sp. FKA-62. We screened for fungal genera that have the ability to induce production of antifungal BE-31405. Surprisingly, production of BE-31405 was induced only by co-culturing $T$. siamensis FKA-61 with a variety of BE-31405-sensitive fungal strains but never with BE-31405-insensitive strains. This is the first report of the induced production of a specific antibiotic active against antibiotic-sensitive fungal strains by co-culturing with them.

The Journal of Antibiotics (2015) 68, 573-578; doi:10.1038/ja.2015.28; published online 25 March 2015

\section{INTRODUCTION}

Co-culture is a relatively new and promising method to induce the creation of novel microbial metabolites, as well as for increasing the productivity of known metabolites. ${ }^{1-3}$ We have already reported the discovery of secopenicillide $\mathrm{C}$, together with four known compounds, penicillide, MC-141, pestalasin A and stromemycin, from a co-culture broth of two fungal strains, Penicillium pinophilum (current name; Talaromyces pinophilus) FKI-5653 and Trichoderma harzianum FKI-5655. ${ }^{4}$ While searching for useful fungal metabolites using the co-culture method, we found that a combination of Talaromyces siamensis FKA-61 with Phomopsis sp. FKA-62 resulted in the production of the antifungal agent, BE-31405, ${ }^{5}$ which displays activity against FKA-62. Moreover, we found a wide range of fungal taxa, which could induce the production of BE-31405, when co-cultured with T. siamensis FKA-61. We report here the characterization of fungal metabolites, including BE-31405, isolated from a co-culture broth of two fungal strains, Talaromyces siamensis FKA-61 and Phomopsis sp. FKA-62, as well as the activities of BE-31405 against fungi having the ability to induce production of BE-31405.

\section{RESULTS}

Identification of BE-31405, produced by co-culture of $T$. siamensis with Phomopsis sp. FKA-62, as an antifungal agent

Talaromyces siamensis FKA-61 and Phomopsis sp. FKA-62 were cocultured on potato dextrose agar (PDA, Difco Laboratories, Detroit, MI, USA), where the colony of FKA-62 could not approach that of
FKA-61 (Figure 1). Production of black pigments (probably melanin) was observed at the face of FKA-62 near to the border with FKA-61. Black pigment did not appear in pure culture of either organism.

On the liquid culture in a 500-ml Erlenmeyer flask containing potato dextrose broth (Difco), orange pigments were produced by coculture. Conversely, dark red and yellow pigments were produced during singlar pure culture of FKA-61 and FKA-62, respectively (Figure 2, center).

The three culture broths (pure culture of FKA-61, and of FKA-62, plus the co-culture of FKA-61 with FKA-62) added to same volume of EtOH were evaluated for antifungal activity against both FKA-61 and FKA-62 using the paper disc method (Table 1). Only the co-culture broth showed activity against FKA-62. This result strongly indicated that production of antifungal agents by FKA-61 was induced as a result of co-culture with FKA-62. This co-culture broth also had selective antifungal activity against Mucor racemosus.

Therefore, we proceeded to purify a co-culture broth guided by antifungal activity against FKA-62. As a result, we identified BE-31405 (Figure 3) ${ }^{5}$ as a antifungal agent. BE-31405 showed potent antifungal activity against FKA-62 (inhibition zone: $21 \mathrm{~mm}$ per $10 \mu \mathrm{g}$ ), without activity against FKA-61, even at $100 \mu \mathrm{g}$ (Tables 1 and 2).

Finally, metabolite profile analysis by HPLC confirmed that production of BE-31405 was induced by co-culturing FKA-61 with FKA-62 (Figure 4). BE-31405 was detected only in the co-culture broth, with the productivity of around $170 \mathrm{mgl}^{-1}$. In comparison, the productivities of five other metaboliotes (sclerotinin $\mathrm{A},{ }^{6}$ griseofulvin, ${ }^{7}$

\footnotetext{
${ }^{1}$ Kitasato Institute for Life Sciences, Kitasato University, Tokyo, Japan and ${ }^{2}$ Graduate School of Infection Control Sciences, Kitasato University, Tokyo, Japan ${ }^{3}$ These authors contributed equally to this work.

Correspondence: Dr K Nonaka or Professor S Ōmura, Kitasato Institute for Life Sciences, Kitasato University, 5-9-1 Shirokane, Minato-ku, Tokyo 108-8641, Japan. E-mail: ken@lisci.kitasato-u.ac.jp or omuras@insti.kitasato-u.ac.jp

Received 10 November 2014; revised 21 February 2015; accepted 24 February 2015; published online 25 March 2015
} 


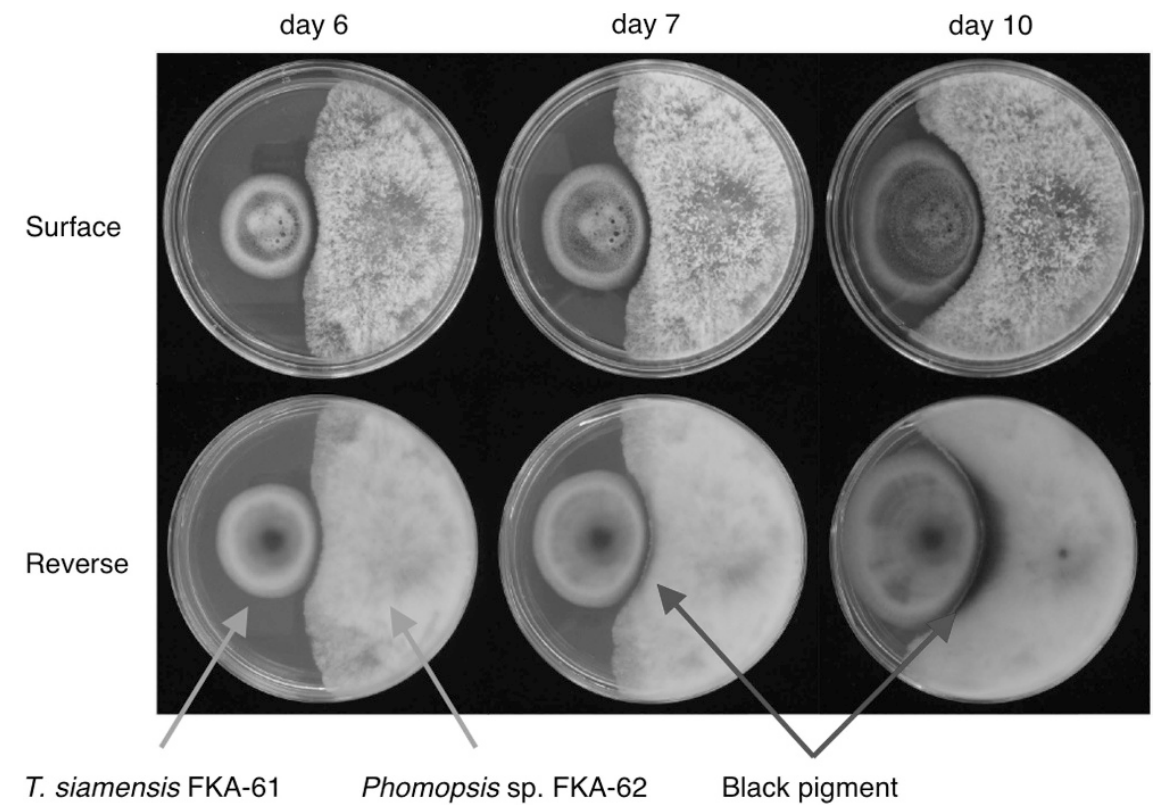

Figure 1 Co-culture of two fungal strains, Talaromyces siamensis FKA-61 and Phomopsis sp. FKA-62, grown on potato dextrose agar (PDA) at $25^{\circ} \mathrm{C}$ for 6,7 and 10 days. A full color version of this figure is available at The Journal of Antibiotics journal online.

vermistatin, ${ }^{8}$ chrodrimanin $\mathrm{F}^{9}$ and chrodrimanin $\mathrm{E}^{9}$ ) produced by FKA-61 in pure culture were not affected by co-culturing with FKA-62. Therefore, we confirmed that the antifungal agent BE-31405 was induced by co-culturing the two strains, FKA-61 and FKA-62.

To reveal which strain assumes the majority under co-culture conditions, a loopful of each co-culture broth of FKA-61 with FKA-62 from day 1 to day 7 during incubation was spread on a PDA plate, followed by incubation at $27^{\circ} \mathrm{C}$ for 7 days (Figure 2, left). As a result, FKA-61 grew dominantly from day 1, despite the growth of FKA-62 being generally faster than that of FKA-61 on PDA (Figure 1). This suggests that, in co-culture, FKA-61 is the dominant strain, probably due to the antifungal effect of BE-31405 produced by FKA-61.

To clarify whether BE-31405 is produced by co-culture in the absence of any direct contact between the two strains, co-culture was performed using a dialysis culture flask (Figure 2, right). As a result, BE-31405 was not detected. This result suggests that direct contact between the two distinct strains is necessary to induce production of BE-31405.

\section{Identification of FKA-61 as a BE-31405-producing strain}

In a co-culture, a colony of FKA-62 did not approach one of FKA-61. Additionally BE-31405 was active against FKA-62 but not against FKA-61. Therefore, we predicted that the strain producing an antifungal factor was likely to be FKA-61. To validate this hypothesis, we conducted co-culturing of FKA-61 or FKA-62 with eight other fungal strains, each belonging to quite different families and orders. Surprisingly, we could detect production of BE-31405 in co-cultures of FKA-61 with six of the fungal strains tested (Table 2). However, FKA-62 did not produce BE-31405 with any strains. This confirmed that FKA-61 is the strain that produces BE-31405.

\section{Antifungal activity of BE-31405 against fungal strains with or} without the ability to induce production of BE-31405 by FKA-61 We evaluated the antifungal activity of BE-31405 against nine fungal strains, irrespective of whether co-culturing with FKA-61 produced
BE-31405. As shown in Table 2, BE-31405 showed antifungal activity against only those six of the strains tested that were able to induce production of BE-31405 by FKA-61. It was not active against the two strains that did not induce FKA-61 to produce BE-31405. This suggests that FKA-61 can produce BE-31405 to attack other fungi that are sensitive to BE-31405 by somehow detecting the presence of such fungi.

\section{DISCUSSION}

A co-culture method was effective in stimulating the generation of novel microbial metabolites and increasing the productivity of known metabolites. However, only specific fungal combinations displayed these characteristics. In a previous study, we reported that metabolites production, including novel productivity of Penicillium pinophilum FKI-5653, was enhanced by co-culturing with Trichoderma harzianum FKI-5655. ${ }^{4}$ At the same time, red pigment was induced, or much increased, by co-culture on an agar plate. In another study, we observed that a novel yellow pigment was produced during co-culture of two different species of the genus Trichoderma on an agar plate as well as in liquid culture. ${ }^{10}$ Exploiting these phenomena, we screened co-cultured fungal combinations on an agar plate using pigment production as an indicator.

Two fungal strains, Talaromyces siamensis FKA-61 and Phomopsis sp. FKA-62, produced a black pigment in the vicinity of the boundary of the adjoining colony when cultured on an agar plate. Moreover, strain FKA-61 inhibited the growth of strain FKA-62. In an earlier report of co-culturing of fungal strains, enhancement of pigment productivity of Monascus occurred as a result of co-culture with either Saccharomyces cerevisiae or Aspergillus oryzae. ${ }^{11}$ Further experiments revealed that hydrolytic enzymes (for example, amylase, chitinase and protease) from a variety of fungi were effective in enhancing Monascus pigment production. ${ }^{11}$ The authors suggested that hydrolysis of Monascus cell walls stimulates a defense mechanism resulting in overproduction of hydrophobic substances, such as pigments, in order to block enzyme attack. ${ }^{11}$ Melanins also protect fungi from hydrolytic enzymes, UV, solar or gamma radiation, extreme temperatures, heavy 


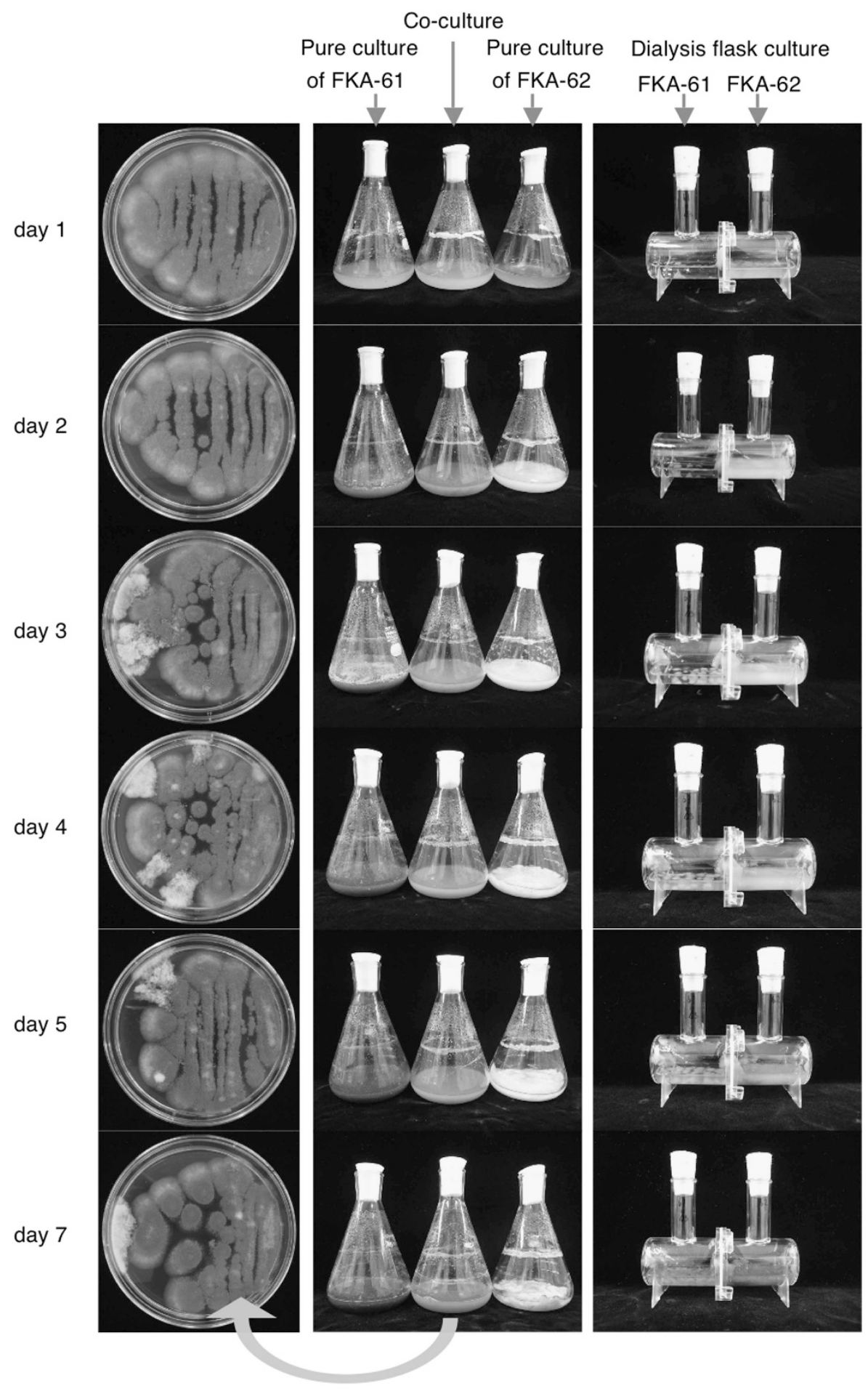

Figure 2 Pure culture and co-culture of two fungal strains, Talaromyces siamensis FKA-61 and Phomopsis sp. FKA-62, grown on potato dextrose broth (PDB) in a 500-ml Erlenmeyer flask at $27^{\circ} \mathrm{C}$ for $1-7$ days (center) and on PDB in a dialysis-culture flask at $27^{\circ} \mathrm{C}$ for $1-7$ days (right) and $1-7-$ day-old co-culture broth grown on potato dextrose agar (PDA) at $25^{\circ} \mathrm{C}$ for 7 days (left). A full color version of this figure is available at The Journal of Antibiotics journal online.

metals and several other toxic compounds. ${ }^{12}$ Similarly, Phomopsis sp. FKA-62 also had the potential to produce black pigments (probably melanin), apparently in order to protect against the antifungal compound BE-31405.

We discovered that a co-culture broth of Talaromyces siamensis FKA-61 and Phomopsis sp. FKA-62 was potently inhibitive of FKA-62, but pure culture broths produced no such activity. We also identified
BE-31405 as an antifungal agent. When strains FKA-61 and FKA-62 were co-cultured on a PDA plate, FKA-62 was not seen to approach FKA-61. Furthermore, BE-31405 was active against FKA-62 but not active against FKA-61. We therefore predicted that FKA-61 was capable of producing BE-31405. For validation of this hypothesis, FKA-61 and FKA-62 were co-cultured with eight different fungal strains. Only strain FKA-61 produced BE-31405 when co-cultured 
Table 1 Antimicrobial activity of culture broths

\begin{tabular}{|c|c|c|c|}
\hline \multirow[b]{2}{*}{ Test organisms } & \multicolumn{3}{|c|}{ Inhibition zone diameter ( $\mathrm{mm}$ ) } \\
\hline & $\begin{array}{c}\text { Pure culture } \\
\text { broth of FKA-61 }\end{array}$ & $\begin{array}{c}\text { Co-culture } \\
\text { broth }\end{array}$ & $\begin{array}{c}\text { Pure culture } \\
\text { broth of } \\
\text { FKA-62 }\end{array}$ \\
\hline Talaromyces siamensis FKA-61 & - & - & - \\
\hline Phomopsis sp. FKA-62 & - & 22 & - \\
\hline Mucor racemosus IFO 4581 & - & 16 & 14 \\
\hline Bacillus subtilis ATCC 6633 & - & - & 14 \\
\hline Kocuria rhizophila ATCC 9341 & - & - & 19 \\
\hline Escherichia coli NIHJ & - & - & - \\
\hline $\begin{array}{l}\text { Xanthomonas camestris pv. } \\
\text { oryzae KB } 88\end{array}$ & - & - & - \\
\hline
\end{tabular}

Culture broth: $50 \mathrm{ml}$ per $8 \mathrm{~mm}$ disc. '-' indicates no inhibition. with six of the eight strains tested. In contrast, FKA-62 did not produce this compound, on its own or in co-culture with any fungal strains.

When strains FKA-61 and FKA-62 were co-cultured on a PDA plate, FKA- 62 produced a black pigment. However, when these strains were co-cultured in liquid medium, an orange pigment was produced. When a co-culture broth was grown on a PDA plate, strain FKA-61 grew relatively better from day 1, even though FKA-62 generally grows faster than FKA-61. Moreover, strain FKA-62 produced a visible black pigment on day 6 . In liquid medium, FKA-62 does not produce black pigment. In addition, BE-31405 was detected from day 1 of a coculture broth by HPLC (data not shown). This clearly suggests that strain FKA-61 was capable of detecting and suppressing FKA-62 by producing the antifungal BE-31405 at a very early stage.

BE-31405 was active against six fungal strains that possessed the ability to induce BE-31405 production when co-cultured with strain FKA-61. It would therefore appear that strain FKA-61 is capable of<smiles>Cc1c(O)c(C)c2c(c1O)C(=O)OC(C)(O)C2C</smiles>

Sclerotinin A

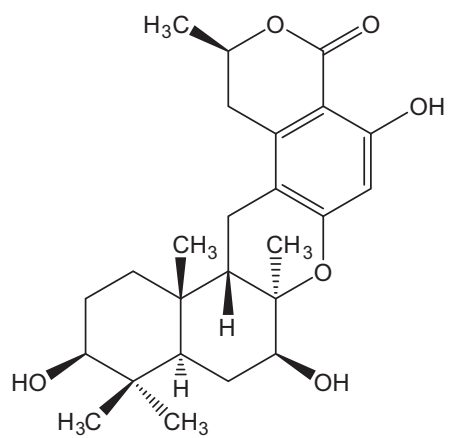

Chrodrimani F<smiles>COC1=CC(=O)C[C@H](C)[C@]12Oc1c(OC)c(OC)cc(OC)c1C2=O</smiles>

Griseofulvin<smiles>C[C@@H]1Cc2c3c(cc(O)c2C(=O)O1)[C@H](O)CC(=O)C(C)(C)[C@@H]1C[C@H](O)[C@@]2(C)O[C@@]1(C)[C@H]2C3</smiles>

Chrodrimanin E<smiles>C/C=C/c1cc(=O)c([C@H]2OC(=O)c3cc(OC)cc(OC)c32)co1</smiles>

Vermistatin<smiles>CC(=O)O[C@H]1c2c(cc(O)c3c2C[C@@]2(C)[C@@]4(C)C=CC(=O)C(C)(C)[C@@H]4C[C@@H](O)[C@@]2(C)O3)C(=O)O[C@@H]1C</smiles>

Thailandolide B<smiles>COc1c(C)cc(CO)c2c1C(=O)OC2O</smiles>

Dihydrogladiolic acid

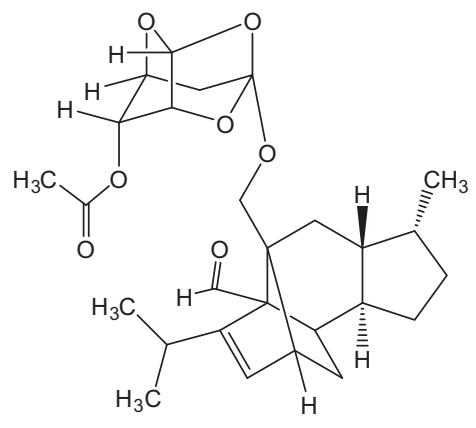

BE-31405

Figure 3 Structures of sclerotinin A, griseofulvin, vermistatin, chrodrimanin F, chrodrimanin E, thailandolide B, dihydrogladiolic acid and BE-31405. 
Table 2 Detection and antifungal activity of BE-31405

\begin{tabular}{|c|c|c|c|c|c|c|c|c|c|}
\hline \multirow[b]{2}{*}{ Order } & \multirow[b]{2}{*}{ Family } & \multirow[b]{2}{*}{ Genus } & \multirow[b]{2}{*}{ Species } & \multirow[b]{2}{*}{ Strain } & \multirow{2}{*}{$\begin{array}{l}\text { Detection of } \\
B E-31405^{a} \\
\text { by co-culture } \\
\text { with FKA-61 }\end{array}$} & \multirow{2}{*}{$\begin{array}{l}\text { Detection of } B E-31405^{a} \\
\text { by co-culture with } \\
\text { FKA-62 }\end{array}$} & \multicolumn{3}{|c|}{ Inhibition zone $(\mathrm{mm})^{\mathrm{b}}$} \\
\hline & & & & & & & $100 \mu g$ & $10 \mu g$ & $1 \mu g$ \\
\hline Eurotiales & Trichocomaceae & Talaromyces & siamensis & FKA-61 & Not tested & Detected & - & - & - \\
\hline Diaporthales & Diaporthaceae & Phomopsis & grabrae & FKI-6685 & Detected & Not detected & 27 & 11 & - \\
\hline Xylariales & Amphisphaeriaceae & Pestalotiopsis & foedans & FKI-6651 & Detected & Not detected & 29 & 16 & - \\
\hline \multirow[t]{3}{*}{ Hypocreales } & Hypocreaceae & Trichoderma & harzianum & FKI-5655 & Not detected & Not detected & - & - & - \\
\hline & Nectriaceae & Mariannaea & elegans & FKI-6644 & Detected & Not detected & 25 & 10 & - \\
\hline & Ophiocordycipitaceae & Purpreocillium & lilacinum & FKI-4665 & Detected & Not detected & 14 & - & - \\
\hline Sordariales & Chaetomiaceae & Staphylotrichum & boninense & FKI-4751 & Detected & Not detected & 31 & 13 & - \\
\hline
\end{tabular}

'-' indicates no inhibition.

a'Detection of BE-31405 by HPLC.

${ }^{b}$ Antifungal activity of BE-31405 against a fungal strain.

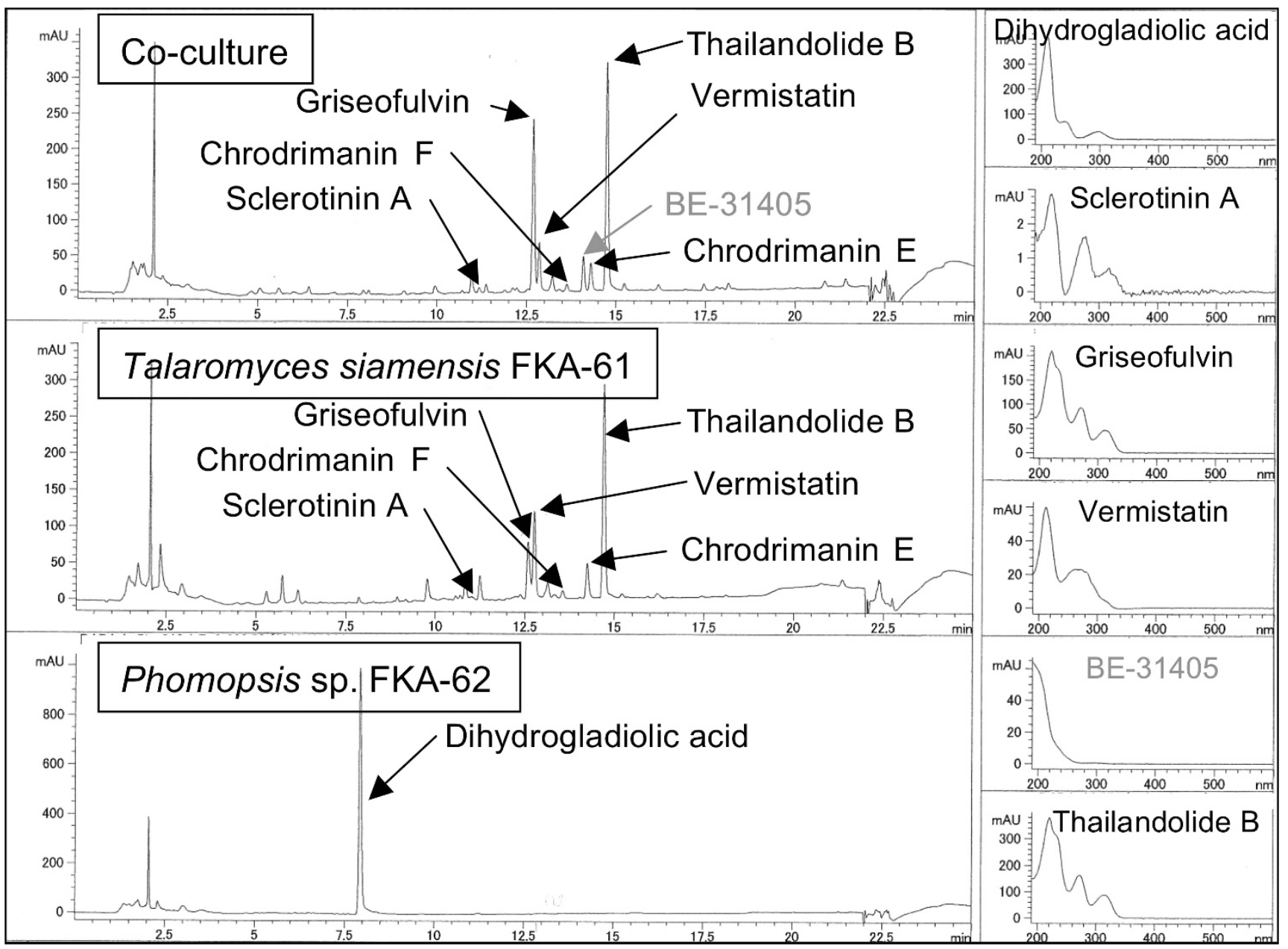

Figure $4 \mathrm{HPLC}$ chromatograms of $50 \% \mathrm{EtOH}$ extract of co-culture and pure culture. The right column indicates UV spectra of sclerotinin A, griseofulvin, vermistatin, BE-31405 and thailandolide B produced by Talaromyces siamensis FKA-61, as well as that of dihydrogladiolic acid by Phomopsis sp. FKA-62. A full color version of this figure is available at The Journal of Antibiotics journal online. 
detecting BE-31405-sensitive fungi and producing the compound to minimize competition. It has been reported that arbuscular mycorrhizal fungi recognize their host plant using signalling molecules (strigolactones and 5-deoxy-strigol) from the root exudates of legume plant Lotus japonicus. ${ }^{13}$ It seems reasonable to assume that strain FKA-61 has the ability to recognize fungi as potential competitors perhaps though similar mechanisms, such as signal compounds. However, co-culture in a dialysis-culture flask did not result in the appearance of BE-31405. Consequently, another mechanism must be responsible, possibly the necessity of direct contact between hyphae of each fungus. Recently, Schroeckh et al. ${ }^{14}$ discovered that intimate physical interaction of Aspergillus nidulans with the soil-dwelling bacterium Streptomyces rapamycinicus specifically activated silent fungal secondary metabolism genes, resulting in the production of the archetypal polyketide orsellinic acid and its derivatives. The Saga/ Ada complex containing the histone acetyltransferase is required for induction of the orsellinic acid gene cluster by the bacterium, and the Saga/Ada-dependent increase of histone 3 acetylation at lysine 9 and 14 occurs during interaction of fungus and bacterium. ${ }^{15}$ In our research, there is a possibility that a similar phenomenon occurred during interaction of the two fungi. The entomopathogenic fungus, Beauveria bassiana, is also capable of recognizing insects. The lectin, a kind of carbohydrate-binding glycoprotein, on the conidial surface of B. bassiana interacts with the insect curticle. ${ }^{16}$ Thus strain FKA-61 may recognize a fungal competitor by a physical difference in hyphal surface structure or hyphal component.

Strain FKA-61 does not take any action against Talaromyces pinophilus FKI-5653 and Trichoderma harzianum FKI-5655. FKI-5653 is of the same genus (Talaromyces) as FKA-61 but is a different species. In a previous study, we found an enhancement of metabolite productivity of Talaromyces pinophilus FKI-5653 when it was cocultured with Trichoderma harzianum FKI-5655. ${ }^{4}$ Furthermore, all metabolites of FKI-5653 were not active against FKI-5655. ${ }^{4}$ This suggest that the genera Trichoderma and Talaromyces can exist alongside each other. Now we are trying to identify the mechanism by which strain FKA-61 is able to recognize other fungal species and how its chemical defenses are triggered. We are also proceeding with co-culture experiments with a view to discover yet more novel antifungal agents.

\section{METHODS}

\section{General}

NMR spectra were measured on a Varian XL-400 spectrometer with ${ }^{1} \mathrm{H}$ NMR at $400 \mathrm{MHz}$ and ${ }^{13} \mathrm{C}$ NMR at $100 \mathrm{MHz}$ (Agilent Technologies, Santa Clara, CA, USA). The chemical shifts are expressed in p.p.m. and are referenced to the solvent $\mathrm{CD}_{3} \mathrm{OD}$ (3.30 p.p.m.) in the ${ }^{1} \mathrm{H}$ NMR spectra and to the solvent $\mathrm{CD}_{3} \mathrm{OD}$ (49.0 p.p.m.) in the ${ }^{13} \mathrm{C}$ NMR spectra. FAB-MS and ESI-MS spectra were measured on a JEOL JMS AX-505 HA mass spectrometer and a JEOL AccuTOF apparatus (JEOL, Akishima, Tokyo, Japan).

\section{Antimicrobial activities}

Antimicrobial activities of culture broths against representative microbes. Antimicrobial activity was measured using the paper disc method $(8 \mathrm{~mm}$, ADVANTEC, Tokyo, Japan). Samples contained $50 \mu \mathrm{l}$ of $50 \%$ EtOH extract of pure culture broths of strains FKA-61 and FKA-62, as well as the co-culture broth. Culture conditions were as follows: Bacillus subtilis ATCC 6633 (Davis synthetic medium $\left(0.7 \% \mathrm{~K}_{2} \mathrm{HPO}_{4}, 0.2 \% \mathrm{KH}_{2} \mathrm{PO}_{4}, 0.5 \%\right.$ sodium citrate, $0.1 \%$ ammonium sulfate, $0.2 \%$ glucose, $0.01 \% \mathrm{MgSO}_{4} \cdot 7 \mathrm{H}_{2} \mathrm{O}$ and $0.8 \%$ agar), $1.0 \%$ inoculation, $37^{\circ} \mathrm{C}, 24 \mathrm{~h}$ ), Kocuria rhizophila ATCC 9341 (Nutrient agar ( $0.5 \%$ peptone, $0.5 \%$ meat extract and $0.8 \%$ agar), $0.2 \%$ inoculation, $37^{\circ} \mathrm{C}$,
$24 \mathrm{~h}$ ), Escherichia coli $\mathrm{NIHJ}$ (Nutrient agar, $0.5 \%$ inoculation, $37^{\circ} \mathrm{C}, 24 \mathrm{~h}$ ), Xanthomonas camestris pv. oryzae KB 88 (Nutrient agar, $1.0 \%$ inoculation, $37^{\circ}$ C, $24 \mathrm{~h}$ ), Mucor racemosus IFO 4581 (GY agar (1.0\% glucose, $0.5 \%$ yeast extract and $0.8 \%$ agar adjusted in $\mathrm{pH} 6.0), 0.3 \%$ inoculation, $27^{\circ} \mathrm{C}, 48 \mathrm{~h}$ ), Talaromyces siamensis FKA-61 (PDA (Difco), 0.5\% inoculation, $27^{\circ} \mathrm{C}, 48 \mathrm{~h}$ ), and Phomopsis sp. FKA-62 (PDA, $0.5 \%$ inoculation, $27^{\circ} \mathrm{C}, 72 \mathrm{~h}$ ).

Antifungal activity of BE-31405 against co-cultured fungal strains. Antifungal activity of BE-31405 against each fungal strain using a co-cultured broth was also measured by the paper disc method ( $8 \mathrm{~mm}$, ADVANTEC). Paper discs contained varying amounts of BE-31405. Culture conditions were: Talaromyces siamensis FKA-61, Phomopsis sp. FKA-62, Phomopsis grabrae FKI-6685, Pestalotiopsis foedans FKI-6651, Trichoderma harzianum FKI-5655, Mariannaea elegans var. punicea FKI-6644, Perpreocillium lilacinum FKI-4665, Talaromyces pinophilus FKI-5653, Staphylotrichum boninense FKI-4751, and Gymnascella aurantiaca FKI-6588 (PDA (Difco) adjusted in $\mathrm{pH} 4.3,0.5 \%$ inoculation, $27^{\circ} \mathrm{C}$, $48-72 \mathrm{~h})$.

\section{ACKNOWLEDGEMENTS}

This study was supported, in part, by funds from the Quality Assurance Framework of Higher Education from the Japan's Ministry of Education, Culture, Sports, Science and Technology (MEXT) and a Kitasato University Research Grant for Young Researchers. We thank Dr. Yukihiro Asami (Kitasato University) for helpful comments on the manuscript and Dr. Kenichiro Nagai (Kitasato University) for measurements of MS.

1 Pettit, R. K. Mixed fermentation for natural product drug discovery. Appl. Microbiol. Biotechnol. 83, 19-25 (2009).

2 Wang, J.-P., Lin, W., Wray, V., Lai, D. \& Proksch, P. Induced production of depsipeptides by co-culturing Fusarium tricinctum and Fusarium begoniae. Tetrahedron Lett. 54, 2492-2496 (2013).

3 Bertrand, S. et al. Detection of metabolite induction in fungal co-cultures on solid media by high-throughput differential ultra-high pressure liquid chromatography-timeof-flight mass spectrometry fingerprinting. J. Chromatogr. A. 1292, 219-228 (2013)

4 Nonaka, K. et al. Enhancement of metabolites productivity of Penicillium pinophilum FKI-5653, by co-culture with Trichoderma harzianum FKI-5655. J. Antibiot. 64, 769-774 (2011).

5 Okada, $\mathrm{H}$. et al. BE-31405, a new antifungal antibiotic produced by Penicillium minioluteum. I. Description of producing organism, fermentation, isolation, physicochemical and biological properties. J. Antibiot. 51, 1081-1086 (1998).

6 Sassa, T., Aoki, H., Namiki, M. \& Munakata, K. Plant growth promoting metabolites of Sclerotinia sclerotiorum. Part I. Isolation and structures of sclerotinin A and B. Agrc. Biol. Chem. 32, 1432-1439 (1968).

7 Grove, J. F., MacMillan, J., Mulholland, T. P. C. \& Rogers, M. A. T. 759 Griseofulvin. Part I. J. Chem. Soc. 3949-3958 (1952).

8 Fuska, J., Uhrín, D., Proksa, B., Votický, Z. \& Ruppeldt, J. The structure of vermistatin, a new metabolite from Penicillium vermiculatum. J. Antibiot. 39, 1605-1608 (1986).

9 Hayashi, H., Oka, Y., Kai, K. \& Akiyama, K. New chrodrimanin congeners, chrodrimanins D-H, from YO-2 of Talaromyces sp. Biosci. Biotechnol. Biochem. 76, 1765-1768 (2012)

10 Kaifuchi, S. et al. Inducing pigmentation using Hypocrea/Trichoderma co-cultures. Jpn J. Mycol. 53, 71-81 (2012).

11 Shin, C. S., Kim, H. J., Kim, M. J. \& Ju, J. Y. Morphological change and enhanced pigment production of Monascus when cocultured with Saccharomyces cerevisiae or Aspergillus oryzae. Biotechnol. Bioeng. 5, 576-581 (1998).

12 Nosanchuk, J. D. \& Casadevall, A. Impact of melanin on microbial virulence and clinical resistance to antimicrobial compounds. Antimicrob. Agents Chemother. 50, 3519-3528 (2006).

13 Akiyama, K., Matsuzaki, K. \& Hayashi, H. Plant sesquiterpenes induce hyphal branching in arbuscular mycorrhizal fungi. Nature 435, 824-827 (2005)

14 Schroeckh, V. et al. Intimate bacterial-fungal interaction triggers biosynthesis of archetypal polyketides in Aspergillus nidulans. Proc. Natl Acad. Sci. USA 106 14558-14563 (2009).

15 Nützmann, H. W. et al. Bacteria-induced natural product formation in the fungus Aspergillus nidulans requires Saga/Ada-mediated histone acetylation. Proc. Natl Acad. Sci. USA 108, 14282-148287 (2011).

16 Sandhu, S. S. et al. Myco-biocontrol of insect pests: factors involved, mechanism, and regulation. J. Pathog. 2012, 126819 (2012).

Supplementary Information accompanies the paper on The Journal of Antibiotics website (http://www.nature.com/ja) 